\title{
Can X-Ray Jets Be Cosmic Beacons?
}

\author{
Dan Schwartz ${ }^{1}$,
}

\begin{abstract}
If X-rays observed from any extragalactic radio jets are due to inverse Compton scattering on the cosmic microwave background $(\mathrm{CMB})$ radiation, then such a source will be detectable with the same surface brightness anywhere in the more distant universe. Chandra observations imply that such systems do exist, and will therefore serve as Cosmic Beacons out to the redshift at which they form.
\end{abstract}

\section{Introduction}

PKS 0637-752, the first celestial X-ray target of the Chandra X-ray Observatory, revealed a surprisingly strong X-ray jet coincident with the previously known radio jet (Schwartz et al. 2000). The great difficulty in explaining the X-ray emission and allowing the magnetic field and relativistic electrons to be near equipartition, led to suggestions (Celotti et al. 2001; Tavecchio et al. 2000) that the extended jet structure was in bulk relativistic motion with $\Gamma \sim 10$ on scales greater than $100 \mathrm{kpc}$. With Chandra detection of X-ray jets soon to be in the tens of objects, models for at least 3C 273 (Sambruna et al. 2001), and 3C 371 (Pesce et al. 2001) also call for large scale relativistic motions.

We point out here that if relativistic beaming is common, we should see X-ray jets from those sources pointed toward us anywhere in the universe. Even powerful unbeamed sources may be seen at all redshifts, if their intrinsic magnetic fields are somewhat less than the typical values $\sim 2 \times 10^{-4}$. This follows from the standard relation that the ratio of IC power to synchrotron power is the ratio of the energy density of target photons to the magnetic field energy density (Felten and Morrison 1966). The CMB energy density increases with redshift according to $(1+z)^{4}$, (Fig. 1). This factor compensates for the decrease of surface brightness due to the expanding universe. X-ray jets are resolved by Chandra and will always be resolved (on-axis) anywhere in the universe (Fig. 2).

As the jets maintain their apparent surface brightness, they can even outshine the quasar cores. Such a case might be speculated for PKS $2215+020$, at $\mathrm{z}=3.572$, where ROSAT detects an unusually high X-ray to optical flux ratio (Siebert and Brinkmann 1998). Other cases should occur among the distant radio emitting Sloan Survey quasars (Fan et al. 2001).

\footnotetext{
${ }^{1}$ Harvard-Smithsonian Center for Astrophysics
} 


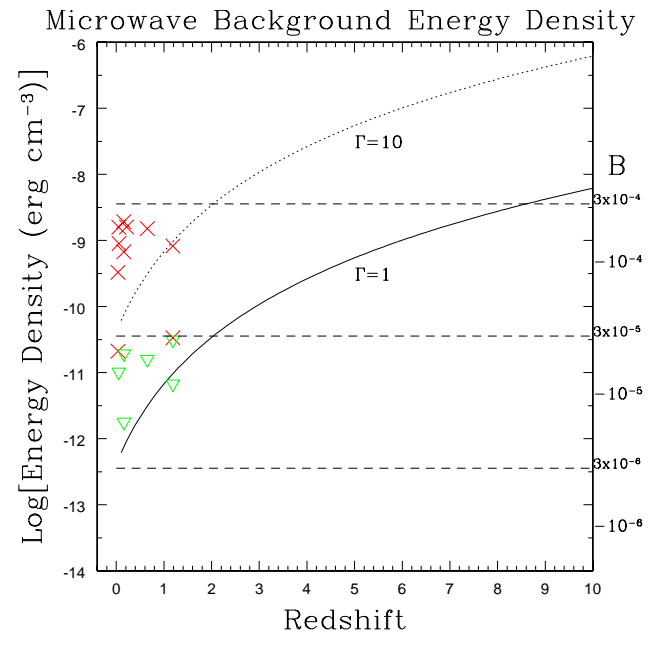

Fig. 1. - The continuous curves give the apparent CMB energy density (on the left vertical scale) vs. redshift as the solid line (in the rest frame of the CMB) or the dotted line (in a rest frame moving with bulk Lorentz factor $\Gamma=10$ with respect to the CMB). The crosses and diamonds plot the redshift and inferred magnetic field (on the right vertical scale) for observed knots and hotspots in X-ray jets. The crosses assume non-relativistic bulk motion, while the diamonds allow the jet to have the necessary bulk Lorentz factor to produce the $\mathrm{X}$-ray emission while maintaining equipartition in its rest frame. (Data from (Hardcastle et al. 2001; Harris and Krawczynski 2002; Pesce et al. 2001; Wilson et al. 2000, 2001))

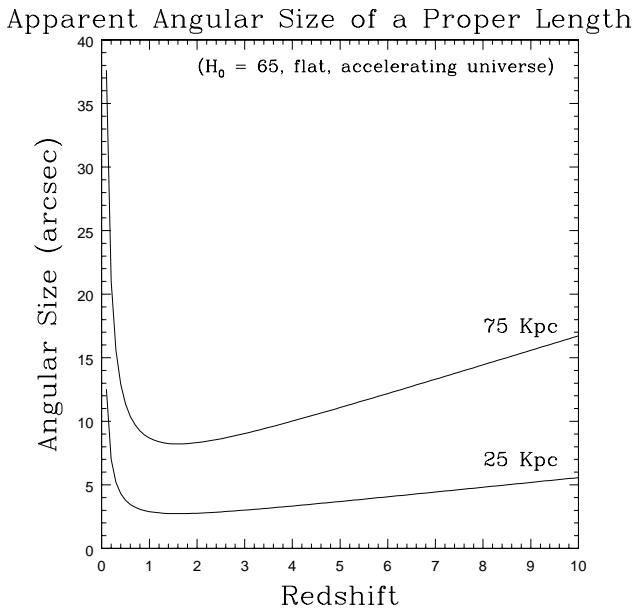

Fig. 2.- The X-ray jets in PKS 0637752 (Schwartz et al. 2000), and 3C 273 (Marshall et al. 2001) brighten about 20 to $30 \mathrm{kpc}$ from the quasar core, and extend 50 to $100 \mathrm{kpc}$ (all distances projected in the plane of the sky). We plot the apparent angular size of projected distances of 25 and $75 \mathrm{kpc}$ as a function of redshift. With the $1^{\prime \prime}$ resolution of Chandra, 10 kpc can be resolved anywhere in the universe. Throughout this paper we assume a flat, accelerating cosmology, with $\mathrm{H}_{0}=65, \Omega_{0}=0.3$, and $\Omega_{\Lambda}=0.7$, and use the formulas given by Pen (1999) 


\section{Observational Implications}

Jets may be displaced by $5^{\prime \prime}$ to $10^{\prime \prime}$ from the optical object which emits them. They may therefore be blank fields within the best location capability of ROSAT, Chandra, and XMM/Newton. They might also be incorrect identifications of chance coincidences with optical objects. Jets require $\leq 1^{\prime \prime}$ angular resolution for clear identification. If resolved, the jet should point toward the AGN. Algorithms to detect extended sources for telescopes with poorer resolution, e.g. when more than $5^{\prime}$ offaxis in Chandra, must fit to a linear shape, not to a circularly symmetric shape, for optimum efficiency.

\section{What Can We Learn?}

- Cosmogony and Jet Physics

- Discover distant, early activity in the universe.

- Verify the common occurrence of relativistic beaming on $100 \mathrm{kpc}$ scales. If this occurs, X-ray jets from such objects are certain to be seen at any redshift at which they exist.

- Is the CMB the limiting dynamical factor in radio lobe and jet formation? The X-rays are produced with electrons of $\gamma \sim 1000 / \Gamma$, which will have lifetimes decreasing from $\sim 10^{8} \Gamma$ to $10^{5} \Gamma$ years, for redshifts $\mathrm{z}=1$ to 10 , where $\Gamma$ is the bulk Lorentz factor of the jet.

- Are jets established before the accretion luminosity in the core becomes significant? (In this case there might be no optical counterpart to an X-ray jet.)

- Are there radio quiet X-ray jets? This could happen if "radio" electrons at higher $\gamma$ have lost their energy to gamma rays, or if larger jet structures can transport upwards of $10^{46} \mathrm{erg} \mathrm{s}^{-1}$ while $B \leq 10^{-5}$ Gauss.

- Do jets produce an appreciable component of the X-ray background? Although the diffuse X-ray background clearly arises from AGN, there are significant implications for producing the observed spectral shape, and for the mix of absorbed and unabsorbed AGN.

\section{- Cosmology}

- Measure the temperature of the cosmic microwave background in situ.

- Verify the decrease of surface brightness according to $(1+z)^{-4}$ (Tolman effect; (Sandage 1961; Sandage and Lubin 2001)). 


\section{Conclusions}

We should vigorously search for distant X-ray jets in both wide field and deep field surveys. Wide field surveys will discover the relatively rare but bright objects such as PKS 0637-752, if the explanation of relativistic beaming with $\Gamma \sim 10$ occurs in Nature. If relativistic beaming is not common, then deep surveys will detect weak X-ray jets from all radio jet sources at redshifts 3 to 10, if they exist!

We acknowledge NASA contract NAS8-39073 to the Chandra X-ray Center.

\section{REFERENCES}

Celotti, A., Ghisellini, G., and Chiaberge, M. 2001, MNRAS, 321, L1

Fan, X. et al. 2001, A.J. 122, in press

Felten, J. E. and Morrison, P. 1966, Ap.J., 146, 686

Hardcastle, M.J., Birkinshaw, M. and Worrall, D.M. 2001, MNRAS, 323, L17

Harris, D. E., and Krawczynski, H. 2002, Ap.J., 565, in press

Marshall, H.L., et al. 2001, Ap.J., 549, L167

Pen, U-L. 1999, Ap.J. Suppl., 120, 49

Pesce, J.E., et al. 2001, Ap.J., 556, L79

Sambruna, et al. 2001, Ap.J., 549, L161

Sandage, A. 1961, Ap.J., 133, 355

Sandage, A. and Lubin, L.M. 2001, A.J., 121, 2271

Schwartz, D. A., et al. 2000, Ap.J., 540, L69

Siebert, J. and Brinkmann, W. 1998, Astron. Astrophys., 333, 63

Tavecchio, F., Maraschi, L., Sambruna, R. M., and Urry, C. M. 2000, Ap.J., 544, L23

Wilson, A.S., Young, A.J., and Shopbell, P.L., 2000, Ap.J., 544, L27

Wilson, A.S., Young, A.J., and Shopbell, P.L., 2001, Ap.J., 547, 740 